\title{
A SIMULATION STUDY OF WiMAX BASED COMMUNICATION SYSTEM USING DELIBERATELY CLIPPED OFDM SIGNAL
}

\author{
Mazid Ishtique Ahmed ${ }^{1}$, Chowdhury Muktadir Rahman', Sabiha Sattar ${ }^{3}$ \\ ${ }^{1}$ Lecturer, Faculty of Science \& Technology, Atish Dipankar University of Science and Technology, Dhaka, Bangladesh \\ ${ }^{2}$ Specialist, PS Core Planning, Robi Axiata Limited, Dhaka, Bangladesh \\ ${ }^{3}$ Engineer, Electronics Division, Bangladesh Atomic Energy Center, Dhaka, Bangladesh
}

\begin{abstract}
WiMAX is a highly sophisticated technology in the broadband wireless access communication system. Scalable Orthogonal Frequency Division Multiple Access (OFDMA) is a key technology behind mobile WiMAX and it is also expected to play a key role in 3GPP Long Term Evolution (LTE) standards. Designers and OEMs have to concentrate on flexibility, scalability and stability of the overall Orthogonal Frequency Division Multiplexing (OFDM) system along with its proper data processing and channeling process to achieve high performance and competitiveness. In this paper, the performance of a strictly band limited OFDM signal is examined using deliberate clipping method, one of the simplest signal distortion based way to reduce high Peak to Average Power Ratio (PAPR) and its effect on the resultant Bit Error Rate (BER) against Signal to Noise Ratio (SNR) performance. A simulation program using MATLAB software was developed to investigate performance of OFDM signal by optimization of different parameters such values of FFT size, Cyclic Prefix co-efficient (CPC) and The Voltage Clipping Ratio (VCR). The simulation results show that with the increment of VCR at optimized parameter values of FFT size and CP, the performance of BER vs SNR improves compared to the results found without clipping.
\end{abstract}

Keywords: WiMAX, PAPR, OFDM, CPC, Voltage Clipping Ratio, Deliberately Clipped

\section{INTRODUCTION TO WIMAX TECHNOLOGY}

Wireless communications have become increasingly popular and broadly required in today's fast paced world, specially for the people of developing countries like Bangladesh. Instant access to virtually unlimited information and resources has become the way of life for individuals in different sector of living and earning their livelihood. Access to various forms of information such as image, data, voice, video and multimedia in an easily communicable, securely and in cost effective manner are the basic requirements of modern day technology savvy society.

Wi-Fi (IEEE Standards based 802.11) has dominated as the most popular wireless access technology within the home and office since 2005 due to its cheaper hardware price, easy to use and interoperability within a range of 30 meters. When used in Metropolitan Area network (MAN) the operation of Wi-Fi started to face challenges for its range, QoS and security. Standard Wi-Fi technology is limited to $100 \mathrm{~m}$ range in Line-of-Sight (LOS) environment which in the pre-release versions of $802.11 \mathrm{n}$ got standardized to $250 \mathrm{~m}$ by the use of MIMO Antenna at both Access Point and The Subscriber Station [1]. Despite all the developments, Wi-Fi remains limited to LAN space in terms of range and depends on WAN technologies to bridge the last miles to access internet and external connectivity.
Broadband Cellular Wireless (BCW) system thus appears as the technique to satisfy this rapidly growing demand of communication systems. A key benefit of it is the ability to use bidirectional antennas which results in improved strength of signal on both directions. The standards $802.16 \mathrm{~d}$ and 802.16e popularly known as WiMAX (Worldwide Interoperability for Microwave Access) become a key technology in mitigating the previous issues of range. Since the late 90s, WiMAX technology based communication system has been addressed y the IEEE802.16 group which was formed in 1998 to develop an air-interface standard for wireless broadband. The original 802.16 standard was based on single carrier physical (PHY) layer with a burst Time Division Multiplexed (TDM) Machine Access Control (MAC) layer. After that the IEEE 802.16 group subsequently produced $802.16 \mathrm{a}$ (an amendment to the standard), to introduce NLOS applications in the $2 \mathrm{GHz} \sim 11 \mathrm{GHz}$ band, using OFDM based PHY layer with addiotnal support of OFDMA in the MAC layer. In December 2005, the IEEE group completed and approved IEEE 802.16e-2005, an amendment which added mobility support and forms the basis for the WiMAX solution for mobile applications referred as mobile WiMAX [2]. And the WiMAX forum has been formed to promote the interoperability between manufacturers and vendors for the conformance to the standard. 


\section{ORTHOGONAL FREQUENCY DIVISION}

\section{MULTIPLEXING (OFDM)}

Mobile WiMAX uses OFDM as a multiple access technique which is a joint process of modulation and multiplexing.

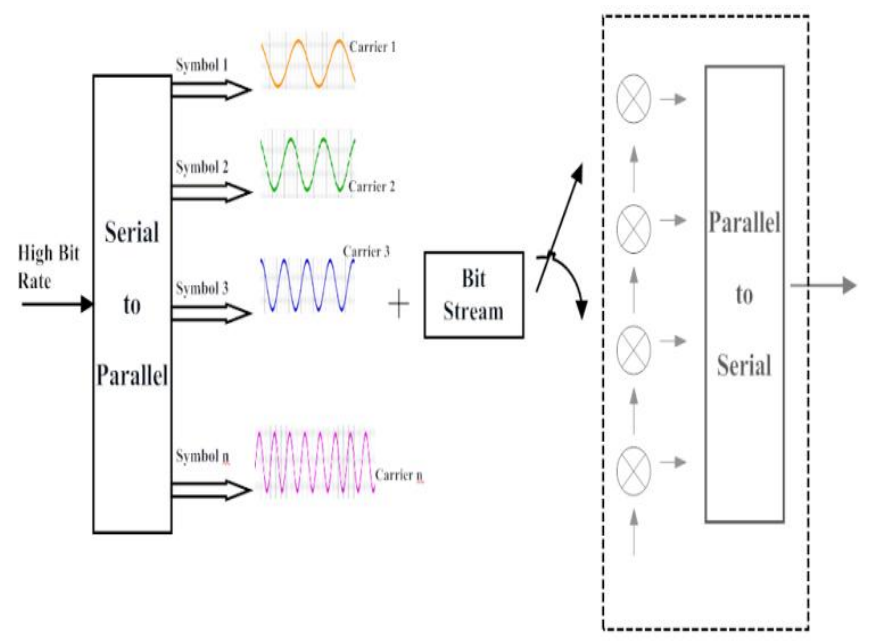

Fig-1: Functional Block Diagram of OFDM Signal Generation

In OFDM, multiplexing operation is performed on independent signals which are a sub-set of the one main signal. The signal first itself is split into independent channels, modulated by data and then multiplexed to create the OFDM carrier.

The main concept in OFDM is Orthogonality of the subcarriers. As all the sub-carriers are sine or cosine wave, area under one period of sine or cosine wave is zero and the area under a sine wave multiplied by its own harmonic is always zero. Thus orthogonality allows simultaneous transmission on a lot of sub-carriers in a restricted or tight frequency space without being interfered with each other. In this way, it is quite similar to the technique adapted in CDMA where codes are used to make data sequences independent (also orthogonal) and allowing many independent users to transmit in same space successfully.

\subsection{Applications and Parameters of real OFDM}

\section{System}

OFDM is a vastly adapted data multiplexing technique for high speed networks and has gradually increased in commercial usage over the last decade. It is now proposed for radio broadcasting such as in Eureka 147 standard and Digital Radio Mondiale (DRM) [3]. OFDM is used for modem/ADSL application where it co-exists with phone line which is called Discrete Multi Tone (DMT). OFDM also forms the basis for the Digital Audio Broadcasting (DAB) standard in European Market [4].
Table -1: Parameters of real OFDM system

\begin{tabular}{|l|l|}
\hline Data rates & 6 Mbps to 48 Mbps \\
\hline Modulation & $\begin{array}{l}\text { BPSK, QPSK, 16 QAM and } \\
\text { 64 QAM }\end{array}$ \\
\hline Coding & $\begin{array}{l}\text { Convolutional concatenated } \\
\text { with Reed Solomon }\end{array}$ \\
\hline FFT Size & $\begin{array}{l}4 \text { with 52 sub-carriers. 48 } \\
\text { for data and 4 for pilots. }\end{array}$ \\
\hline $\begin{array}{l}\text { Sub-carrier Frequency } \\
\text { Spacing }\end{array}$ & $\begin{array}{l}20 \mathrm{MHz} \text { divided by 64 } \\
\text { carriers }\end{array}$ \\
\hline FFT Period & $3.2 \mu \mathrm{sec}$ \\
\hline Guard Duration & $0.8 \mu \mathrm{sec}$ \\
\hline Symbol time & $4 \mu \mathrm{sec}$ \\
\hline
\end{tabular}

\subsection{OFDMA (Orthogonal Frequency Division}

\section{Multiple Access)}

OFDMA is a multi user version of the popular OFDM digital modulation scheme. Multiple Access is achieved by assigning subsets of sub-carriers to individual users. This allows simultaneous low data transmission rate from several users. OFDMA is fast growing in several applications which are the heart of next generation of wireless communication. Apart from WiMAX, it is adapted for the IEEE 802.20 mobile Wireless MAN standard which is commonly referred as MBWA following to the Evolved UMTS Terrestrial Radio Access (E-UTRA). Figure 2-2, illustrates an overview of the Physical Layer (PHY) for WiMAX base-station with scalable OFDMA based on IEEE 802.16e-2005 [5].

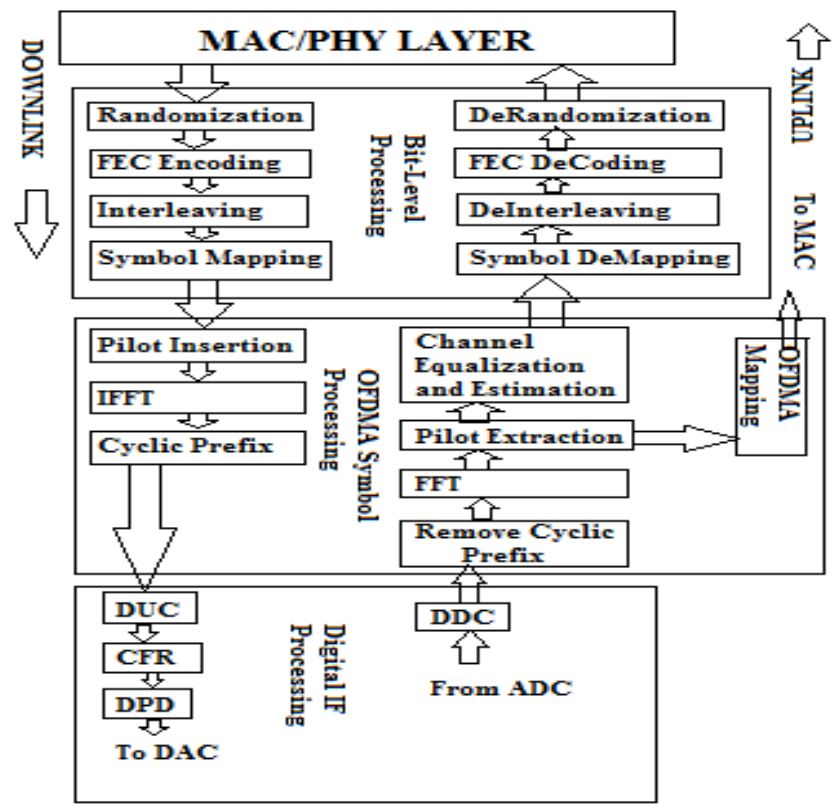

Fig -2: Overview of IEEE 802.16e-2005 Scalable OFDMA Physical Layer for WiMAX Base-stations 


\section{PEAK TO AVERAGE POWER RATIO (PAPR) PROBLEM IN OFDM SYSTEM AND SOLUTIONS}

In advanced mobile communication, OFDM has brought unique features such as high spectral efficiency, robustness to channel fading, immunity to impulse interference and capability of handling very strong multipath fading and frequency selective fading without having to provide power channel equalization [6]. On the other hand, an OFDM signal responses with higher instantaneous peak value with respect to the average signal level causing large amplitude swings when time domain signal travels from a low instantaneous power to high power waveform. As a result, unless the transmitter's power amplifier exhibits an extremely high linearity across the entire signal level range, high Out-Of-Band (OOB) harmonic distortion of power waveform becomes significant which potentially causes interference with adjacent channel. That is why; large Peak to Average Power Ratio (PAPR) is marked as a major issue associated with OFDM signal generation which leads to system performance degradation due to distortions introduced by power amplifiers (PA) or other non-linear devices of the transmitter block.

\subsection{PAPR Definition and Mathematical}

\section{Representation}

PAPR (Peak to Average Power Ratio) is a measurement of waveform, calculated from the peak amplitude of the waveform divided by the RMS value of the waveform. It implies that the PAPR is the maximum instantaneous power normalized by the average power among all possible data patterns. This definition is especially important for the system in which some special coding is employed that has some constraints in the data sequences control the PAPR very low. There thus appear two definitions largely accepted of PAPR for OFDM signal, one of that definition is to assume the PAPR can be expressed in deterministic value [7] [8], that is -

$$
D-P A P R=\sup _{-\infty<t \infty} \zeta(t)=\sup \max _{0 \leq t \leq T_{u}} \zeta_{1}(t)
$$

\subsection{PAPR in OFDM System}

The PAPR in OFDM system increases exponentially with the number of sub-carriers. To evaluate that, let, $\mathrm{A}=[\mathrm{A} 0, \mathrm{~A} 1$, ......., $\mathrm{A}(\mathrm{N}-1)]$ to be modulated data sequences of the length $\mathrm{N}$ during the time interval of $[0, \mathrm{~T}]$, where $\mathrm{Ai}$ is a symbol from a signal constellation and $\mathrm{T}$ is the OFDM symbol duration. Then the complex envelope of the baseband OFDM signal for $\mathrm{N}$ carriers is given by [8],

$$
s(t)=\sum_{n=0}^{N-1} A_{n} \exp \left(j \omega_{0} n t\right)
$$

Where, $\omega_{0}=\frac{2 \pi}{T}$ and $j=p-1$
In practical systems, a guard interval (cyclic prefix) is inserted by the transmitter in order to remove inter-symbol interference (ISI), and inter-channel interference (ICI) in multi-path environment. However in can be ignored since it does not affect PAPR The PAPR of the transmit signal $s(t)$ in equation 2 , is the ratio of the maximum instantaneous power and the average power [9], that is -

$$
\operatorname{PAPR}(\mathrm{A})=\frac{\max |\mathrm{s}(\mathrm{t})|^{2}}{\mathrm{E}\left\{|\mathrm{s}(\mathrm{t})|^{2}\right\}} \ldots \ldots \ldots \ldots \ldots \ldots \ldots \ldots \ldots \ldots
$$

Where, $\mathrm{E}\{$.$\} denotes the expectation operator.$

Usually the continuous time PAPR of $s(t)$ is approximated using the discrete time PAPR shown in equation 3, is obtained from the samples of the OFDM signal.

\subsection{Techniques to reduce PAPR in OFDM system}

Several schemes have been proposed to reduce PAPR. These techniques can mainly be divided into two categories [6]. Those are -

A) The Signal Scrambling techniques are all variations on how to scramble the codes (or modify the phases) to decrease the PAPR. Golay complementary sequences, Shapiro-Rudin sequences, M-sequences, Baker codes can be used to efficiently reduce the PAPR. However, with the increase in the number of carriers, the overhead associated with the exhaustive search or the best code would increase exponentially. More practical solutions of the scrambling mechanism are block coding, selective mapping and partial transmit sequences. Selective mapping and partial transmit sequences are two probabilistic schemes to reduce PAPR.

B) The Signal Distortion techniques reduce higher peaks directly by distorting the signal prior to amplification. Clipping the OFDM signal before amplification is a simple method to limit PAPR. More practical solutions are Peak Windowing, Peak Cancellation, Peak Power Suppression, Weighted Multi-Carrier transmission, Companding and Deliberate Clipping.

\subsection{PAPR Reduction by Deliberate Clipping}

If the number of subcarriers increases however, the peak-toaverage power ratio (PAPR) of the OFDM signal also increases. In many wireless applications, both the peak power efficiency and the bandwidth efficiency are the two most important factors [10]. In strictly band-limited systems, the OFDM signal exhibits a prohibitively high PAPR; and without use of any PAPR reduction technique, efficiency of power consumption at the transmitter becomes very poor. 
The deliberate clipping method may be one of the simplest solutions when the number of sub-carriers is large [11], [12]. The clipping operation causes degradation due to the nonlinear operation, which requires some compensation the deliberate clipping is performed followed by the band pass filter, and the resultant performance degradation is compensated by the $\mathrm{BCH}$ codes [13]. On the other hand, the OFDM signal sampled at the Nyquist rate is clipped and the degradation is compensated by the iterative decision-aided reconstruction of the original OFDM signals [14].

When the clipping is performed on the oversampled OFDM signals, it generally causes the out-of-band radiation of the clipped power, and the band pass filter is required to suppress the out-of-band radiation. The problem of this scheme is the significant PAPR regret due to the band pass filtering [11]. On the other hand, when the clipping is performed on the signals at Nyquist sampling rate, all the distortion noise falls in-band, avoiding the out-of-band radiation due to the deliberate clipping, assuming that the signal is linearly amplified [11], [14].

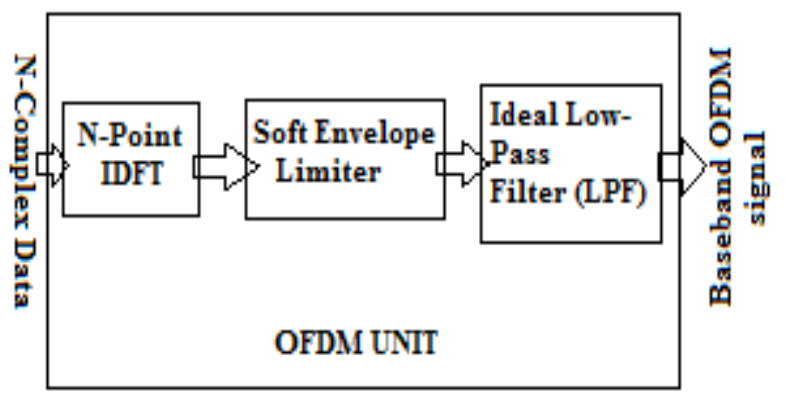

Fig-3: Band Limited OFDM system with Nyquist-rate Clipping

Therefore, in the latter case, the band pass filter for removal of out-of-band radiation is not required. However, probably less recognized is the fact that the ideal low-pass (interpolation) filter (LPF) after clipping illustrated on figure 3, which is necessary for strictly band-limited communications systems, also considerably enlarges the PAPR [15]. Consequently, in such systems the PAPR after the ideal LPF should be taken into account for the evaluation of the PAPR.

Theoretical analysis of the PAPR property of the clipped and low-pass filtered OFDM signals appears quite involved, and thus the PAPR property is studied by extensive computer simulations. In this paper, the definitions and the theoretical remarks that may be useful to describe the PAPR property of the OFDM signals is described.

Let, $s_{n}, n=0,1, \ldots, N-1$ denote the output of the N-point inverse discrete Fourier transformer (IDFT), and let $x(n)$ and $y(n)$ be the real and imaginary parts of $s_{n}$, respectively. Since the input data can be assumed statistically independent and identically distributed, the $\mathrm{x}(\mathrm{n})$ and $\mathrm{y}(\mathrm{n})$ are uncorrelated. Furthermore, for large N, the distribution of both $\mathrm{x}(\mathrm{n})$ and $\mathrm{y}(\mathrm{n})$ approaches Gaussian with zero mean and variance, say $\sigma^{2}$, , due to the central limit theorem. Since the uncorrelated Gaussian random variables are statistically independent $x(n)$ and $y(n)$ are orthogonal, and $x(n)$ and $y(n)$ can be assumed, at least asymptotically, statistically independent. Thus, in the following, it is assumed that the $\mathrm{x}(\mathrm{n})$ and $\mathrm{y}(\mathrm{n})$ are Gaussian random variables.

As a clipping model of the baseband signal, the soft envelope limiter block shown in figure 3 is considered as the function of clipping; the output sample is thus given by -

$$
\widetilde{r_{n}} \triangleq g\left(r_{n}\right)=\left\{\begin{array}{c}
r_{n} ; r_{n} \leq A_{\max } \\
A_{\max } ; r_{n}>A_{\max }
\end{array}\right.
$$

Where, $\widetilde{r_{n}} \triangleq|s(n)|=\sqrt{\left(x_{n}^{2}+y_{n}^{2}\right)}$ is the amplitude of the nth sample of complex OFDM signal and $A_{\max }$ is the maximum permissible amplitude over which the signal is clipped.

The clipping ratio $\gamma$ is defined as,

$$
\gamma \triangleq \frac{A_{\max }}{\sqrt{P_{\text {in }}}}=\frac{A_{\max }}{\sqrt{2 \sigma}}
$$

Where, $\mathbf{P}_{\text {in }}=2 \sigma^{2}$ is the input power of the OFDM signal before clipping.

The total output power $\mathrm{P}_{\text {out }}$, which is the sum of the signal and distortion components, is given by,

$$
\begin{array}{r}
P_{\text {out }}=E_{r n}\left[g^{2}\left(r_{n}\right)\right] \text { or, } \\
P_{\text {out }}=\left(1-e^{-\gamma^{2}}\right) P_{\text {in }} \ldots \ldots \ldots \ldots \ldots \ldots \ldots \ldots \ldots \ldots \ldots \ldots
\end{array}
$$

Normalizing the clipped signal by the rms output power, the sample of the output amplitude is redefined as -

$$
r_{n}=\frac{g\left(r_{n}\right)}{\sqrt{P_{\text {out }}}}=\frac{g\left(r_{n}\right)}{\sqrt{\left(1-e^{-\gamma^{2}}\right) P_{\text {in }}}}
$$

It is to be noted that $\gamma$ cannot be zero by the definition [16]. However, considering the amplitude normalized by the rms output power, it can be defined that $\gamma \neq 0$ by its limit as,

$$
\lim _{\gamma \rightarrow 0} r_{n} \leq \lim _{\gamma \rightarrow 0} \frac{A_{\text {max }}}{\sqrt{P_{\text {out }}}}=\lim _{\gamma \rightarrow 0} \frac{\gamma}{\sqrt{\left(1-e^{-\gamma^{2}}\right) P_{\text {in }}}}=1 \ldots
$$

That is, $r_{n}=1$ for all $n$, which corresponds to the hard (constant) envelop limiter. 


\section{SIMULATION RESULTS OF PERFORMANCE} ANALYSIS OF OFDM SIGNAL

In this section, result of the simulation study on the performance is discussed. To study and find the optimizations, an algorithm illustrated in figure 4, was simulated using MATLAB simulation software. The total analysis is divided into two different parts of which discussed in section 4.1 which deals to determination of optimum FFT size and cyclic prefix co-efficient by analyzing the bit error rate vs signal-tonoise ratio curves followed by results enlisted in section 4.2 which shows the difference or improvement of performance of the baseband OFDM signal while voltage clipping ratio is introduced and optimized.

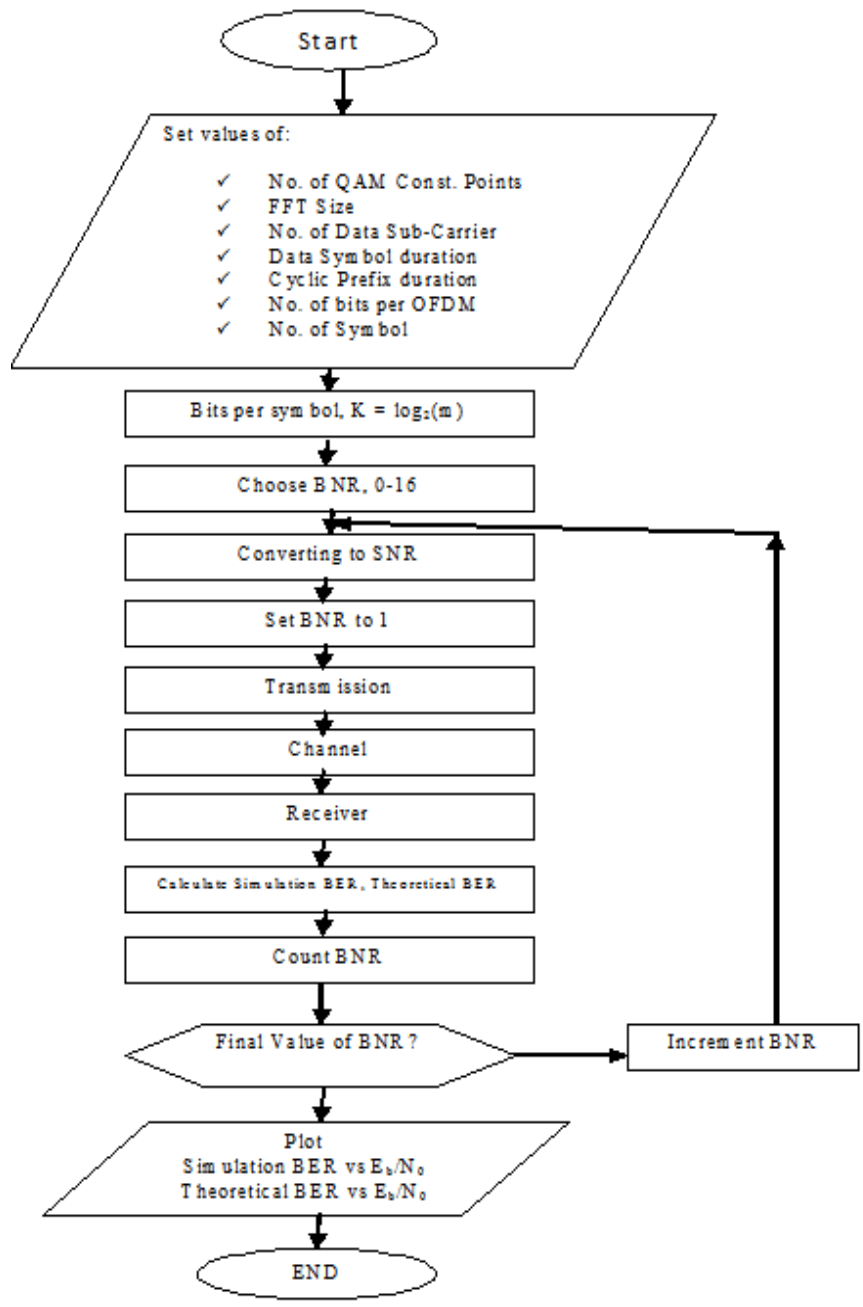

Fig -4: Flow Chart of WiMAX based OFDM Signal for Simulation

\subsection{Optimization of Parameters}

\subsubsection{Determination of Optimum FFT Size}

As shown in the flow chart in figure 4 and also illustrated in figure 1, OFDM signal generation requires the IFFT block to convert the signal from frequency domain to the time domain representation which is essential for estimating the Signal-toNoise Ratio (SNR) of the OFDM signal. The SNR is a function of the FFT size used for the conversion. In the simulation, the performance of the generated OFDM signal is analyzed in terms of BER to SNR by optimizing proper FFT size.

To overcome the problem of the Inter Symbol Interference (ISI) anther parameter is defined as the Cyclic Prefix (CP). For OFDM signal transmission with multiple carriers, $\mathrm{CP}$ is appended. Mathematically, the relation is illustrated below -

$$
\text { Cyclic Prefix Co }- \text { efficeint }=G i=\frac{T_{c p}}{T_{d}} . .
$$

Where, $\mathrm{T}_{\mathrm{cp}}=$ Cyclic Prefix Duration

$$
\mathrm{T}_{\mathrm{d}}=\text { Guard Duration }
$$

Cyclic Prefix Co-efficient or $\mathrm{Gi}$ is the ratio of guard duration to the cyclic prefix duration. While optimizing the FFT size, the Gi value was kept constant to 0.25 .

The values of different parameters used in the simulation for optimization of FFT size is illustrated in table 2. The results are illustrated in

Table-2: Different Parameters used in the simulation for optimization of FFT Size

\begin{tabular}{|l|l|}
\hline \multicolumn{1}{|c|}{ Parameters } & \multicolumn{1}{c|}{ Values } \\
\hline Modulation Scheme & QAM 16 \\
\hline FFT Size & $4,16,64,128$ \\
\hline Data Sub-carriers & 52 \\
\hline Guard Duration & 64 \\
\hline Cyclic Prefix Co-efficient & 0.25 \\
\hline
\end{tabular}

Using the same simulation model, the optimum value of Cyclic Prefix Co-efficient was determined too. Table 3 shows the simulation model parameters for this optimization. For this investigation, the result of the FFT size optimization was kept constant.

Table-3: Different parameters used in simulation for optimization of Cyclic Prefix Co-efficient

\begin{tabular}{|l|l|}
\hline \multicolumn{1}{|c|}{ Parameters } & \multicolumn{1}{c|}{ Values } \\
\hline Modulation Scheme & QAM 16 \\
\hline FFT Size & 64 \\
\hline Data Sub-carriers & 52 \\
\hline Guard Duration & 64 \\
\hline Cyclic Prefix Co-efficient & $0.1,0.2,0.3,0.4,0.5,0.6,0$. \\
& $7,0.8,0.9$ \\
\hline
\end{tabular}




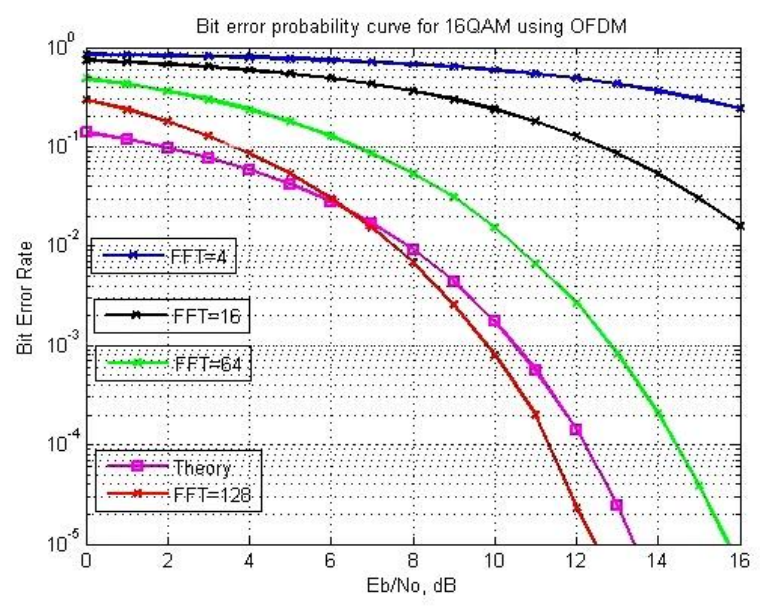

Fig-5: Performance study to optimize FFT Size

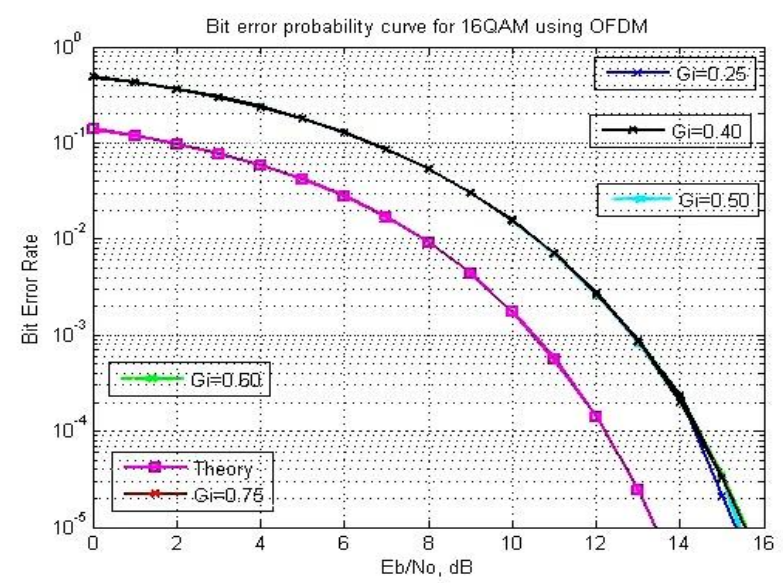

Fig-6: Performance study to optimize Cyclic Prefix

Figure 5 illustrates the simulation results for optimizing FFT size for the baseband OFDM signal using QAM16 modulation technique. As the FFT size was varied through a range of values from 4 to 128 , it was observed that initially the simulation results differed largely compared to theoretical results. When FFT size was increased to 64 , there was significant improvement and for FFT size value 128, it was the near perfect output. But as the FFT size increases, it offers higher complexity in system designing for hardware implementation [18]. That's why the optimum value for FFT Size is determined to be 64 .

Determining the optimization for Cyclic Prefix Co-efficient, Gi the FFT size obtained by the illustration in Figure 5 was fixed at 64 and the similar simulation model was used. The Gi values were varied from 0.1 to 0.9 . The analysis is illustrated on figure 6 where it is visually clear that BER to SNR curve improves with the increase to the value of $\mathrm{Gi}$. The best optimizations were found in a range of 0.4 to 0.7 and for $\mathrm{Gi}=0.5$, the results were closer to that of the theoretical values. As a result, the Cyclic prefix Co-efficient was best kept at $\mathrm{Gi}=0.5$ for optimum performance.

\subsubsection{Determination of Optimum Voltage Clipping Ratio (VCR)}

The main objective of this paper is to study a special type of signal distortion type PAPR reduction scheme which is Deliberate Clipping which is performed by distorting the signal prior to amplification as illustrated in figure 3 .

A significant advantage of clipping is it makes the transmission power efficient. However extensive clipping may cause large Out-of-Band (OOB) and In-band interference. That's why it is highly required to investigate the clipping and understand its effect on overall performance of the baseband OFDM signal. The modified model for this simulation is illustrated in figure 7.

In the simulated model all the parameters i.e. Modulation, Data Sub-carriers are kept at the IEEE 802.16 standard [19]. The FFT size and Cyclic Prefix co-efficient is set according to the optimum value found in the earlier simulation illustrated in the section 4.1.1

In this paper, a term called "Voltage Clipping Ratio (VCR)" or $\mathrm{C}_{\mathrm{p}}$ which is mathematically it is represented as -

$$
C_{p}=\frac{\left|A_{m}\right|}{A}
$$

Where, $A_{m}=A_{\max }$ or $A_{\min }=$ Maximum or Minimum clipping voltage and $\mathrm{A}=$ Clipping threshold voltage. The value of VCR was varied in a range of 5\% to $40 \%$ and observed in terms of the improvement of BER vs SNR curve with respect to earlier optimized results which were performed without clipping the signal. Table 4 lists the parameters for the modified model of simulation.

Table 4: Parameters to optimize the Voltage Clipping Ratio

\begin{tabular}{|l|l|}
\hline \multicolumn{1}{|c|}{ Parameters } & \multicolumn{1}{c|}{ Values } \\
\hline Modulation Scheme & QAM 16 \\
\hline FFT Size & 64 \\
\hline Data Sub-carriers & 52 \\
\hline Guard Duration & 64 \\
\hline Cyclic Prefix Co-efficient & 0.5 \\
\hline Voltage Clipping Ratio (VCR) & $5 \%-40 \%$ \\
\hline
\end{tabular}




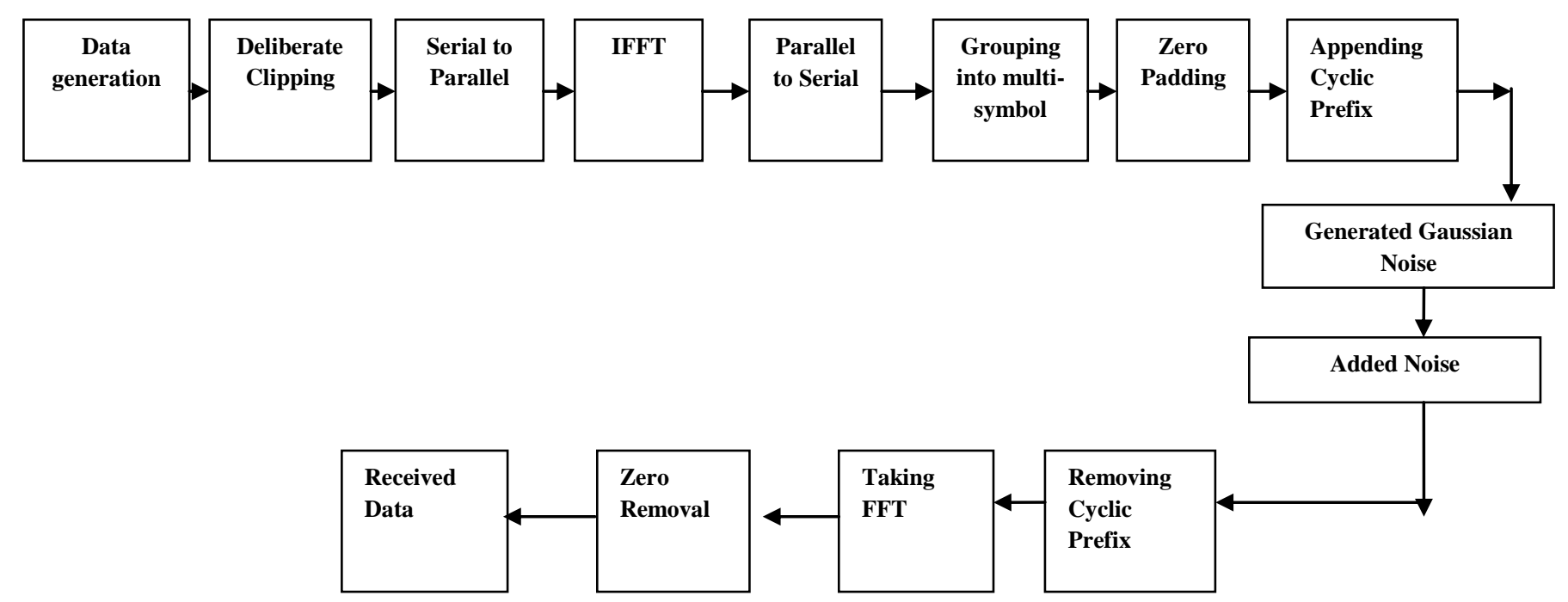

Fig 7: Modified Block diagram of WiMAX based OFDM signal using Deliberate clipping

The results of simulation found by increasing VCR are shown in figures 8, 9, 10, 11, 12 and 13 .

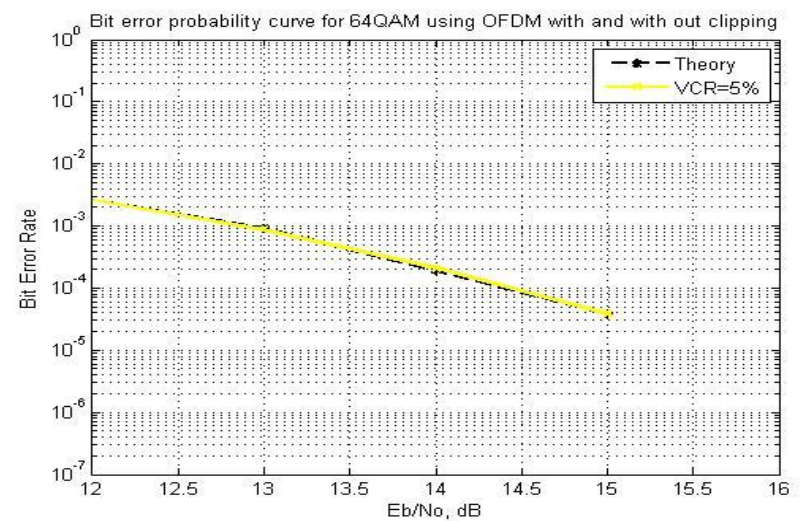

Fig 8: Performance of Clipped OFDM signal for $\mathrm{VCR}=5 \%$

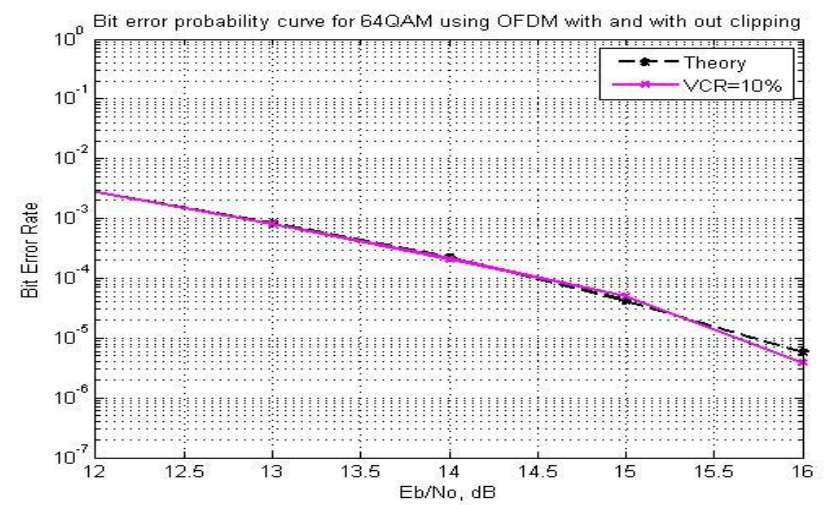

Fig 9: Performance of Clipped OFDM signal for VCR=10\%

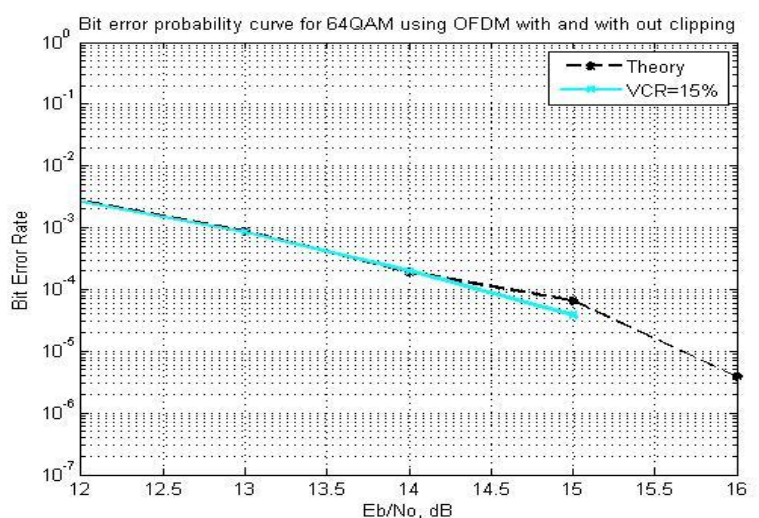

Fig 10: Performance of Clipped OFDM signal for $\mathrm{VCR}=15 \%$

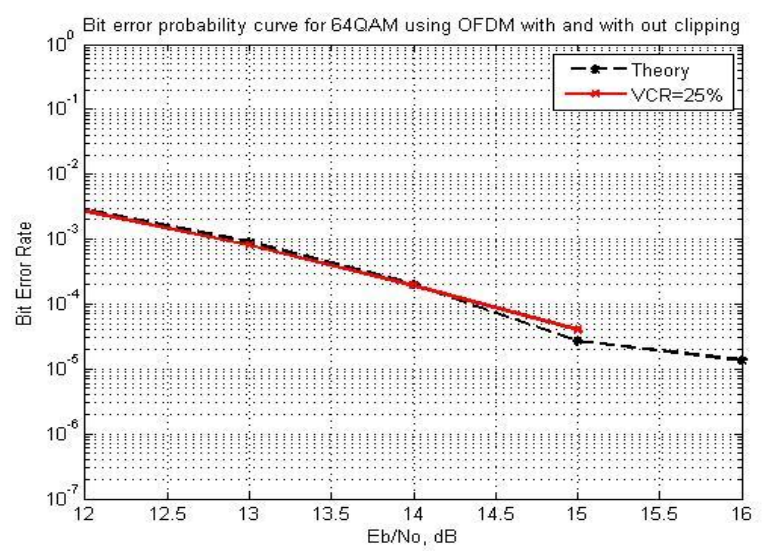

Fig 11: Performance of Clipped OFDM signal for VCR $=25 \%$ 


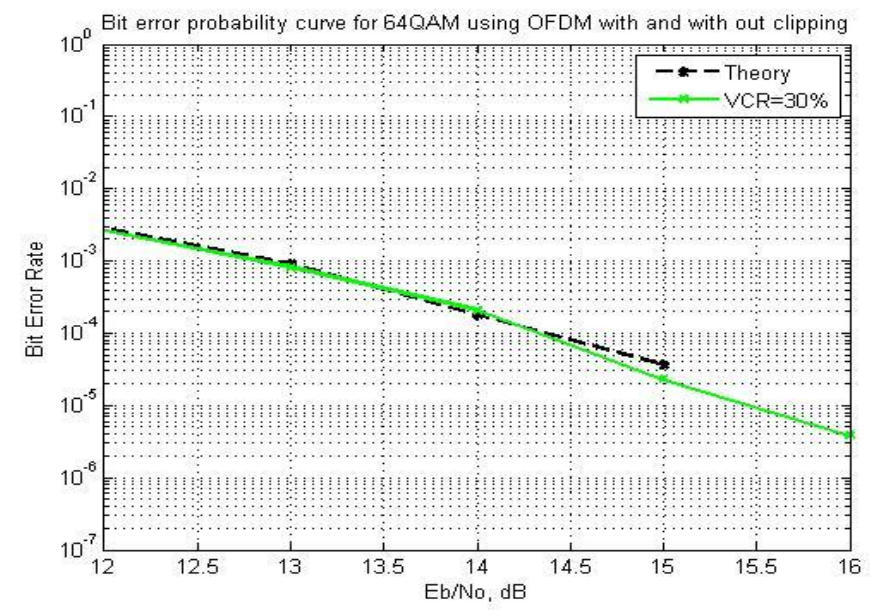

Fig 12: Performance of Clipped OFDM signal for $\mathrm{VCR}=30 \%$

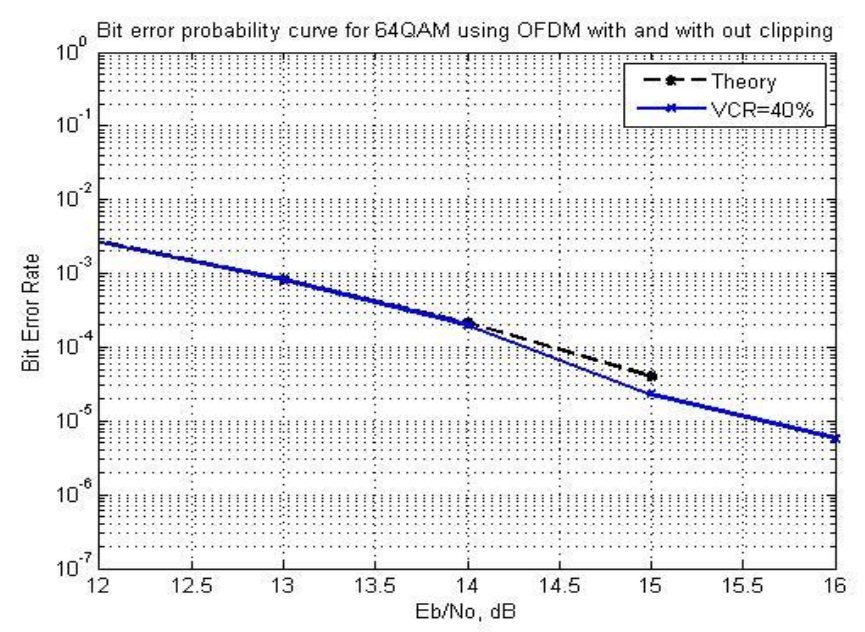

Fig 13: Performance of Clipped OFDM signal for $\mathrm{VCR}=25 \%$

The clipping ratio is kept $5 \%$ in figure 8 and it can be seen from the simulated result the performance has not increased from the without clipped signal. When the clipping ratio is increased to $10 \%$ in figure 9 the simulated results shows much improvement than the 5\% clipping ratio. In figure 10, clipping ratio has been increased to $15 \%$ but the performance is declining. As with $25 \%$ clipping ratio in figure 11 , the performance in most of the cases is same as without clipping result with no improvement or increased performance. But in figure 12 at $30 \%$ clipping ratio, the result is much improved from without clipping and in some cases almost same as without clipping. And finally at $40 \%$, there is deviation from the much improved results obtained for VCR at $30 \%$. So, from the simulated result it is obvious that the most optimum clipping ratio for a wide range of SNR would be 30\%. Which gives a better performance for the simulated BER - SNR and at the same time would also reduce the PAPR making it the most optimum clipping ratio.

\section{CONCLUSIONS}

The nonlinear distortion effects in OFDM transmission have been investigated in many research works. An analytical expression for the output autocorrelation function based on HPA modeling with Bessel series expansion is derived. By using Fourier transformation, the output autocorrelation function can provide information on the power spectral density (PSD) at the HPA output, and at the same time, it allows the analytical calculation of the power of the nonlinear distortion noise [21]. The orthogonal polynomials provide an intuitive means of spectral re-growth analysis and enable us to derive a very simple analytical expression for the autocorrelation function and hence the PSD of the nonlinear noise. In this way, it is possible to derive an analytical BER expression for the OFDM systems performance in presence of nonlinear HPA in AWGN channels [20].

In this paper, in terms of observing the performance of OFDM based WiMAX system, we limited our study within reducing PAPR and improving the BER to SNR of OFDM signal by Deliberate Clipping technique. On the other hand, the OFDM signals are highly sensitive to non linear distortions introduced by the High Power Amplifier (HPA). It has two effects on transmission the first one, is the spectral re-growth of the signal which leads to adjacent channel interference (ACI) and the second one is the effect in the distortion of the signal in the nominal frequency band which causes inter-symbol interference (ISI).

Investigations in these directions are great attempts to optimize OFDM performance enhancement and it is a matter of utter satisfaction to conclude with the observations made throughout the research work.

\section{REFERENCES}

[1]. http://standards.ieee.org/findstds/standard/802.11n2009.html

[2]. "IEEE 802.16 WirelessMAN Standard: Myths and Facts".,http://www.ieee802.org/16/docs/06/C80216-

06_007r1.pdf. Retrieved 2008-03-12

[3]. Ranjan Parekh. "Principles of Multimedia". Tata-McGraw Hill publishing company limited, 2006.

[4]. European Broadcasting Union (EBU). "Digital Radio Mondiale (DRM); Distribution and Communications Protocol (DCP)". ETSI TS 102821 V1.2.1 (2005-10)

[5]. ALTERA, Application note 412. "A Scalable OFDMA Engine for WiMAX”, ver 2.1, May 2007

[6]. Rakesh Rajbansh, Anupama Veeragandham. "OFDM System Design" December 9, 2004

[7]. V. Tarokh and H. Jafarkhani. "On the computation and reduction of the peak-to-average power ratio in multicarrier communications" IEEE Trans. Commun., vol 48, no. 1, pp. 37-44, January 2000

[8]. K.G. Paterson and V. Tarokh. "On the existence and construction of good codes with low peak-to-average power 
ratios.”. IEEE Trans. Inform. Theroy, vol 46, no. 6, pp. 19741987, September 2000

[9]. Tellambura, C. "Computation of the continuous PAPR of an OFDM signal with BPSK sub-carriers". IEEE Communications Letter, vol. 5, May 2001

[10]. S.L. Miller and R.J. O’Dea. "Peak power and bandwidth efficient linear modulation". IEEE Trans. Commun., vol. 46, pp. 1639-1648, December 1998

[11]. R. O'Neill and L.B. Lopes. "Envelope variations and spectral splatter in clipped multi-carrier signals" Pp. 71-75, In Proc. PIMRC'95, Toronto, Canada, September 1995

[12]. X. Li and L. J. Cimini Jr.. "Effects of filtering and clipping on the performance of OFDM" Pp. 1634-1638, In Proc. VTC'97, Phoenix, AZ, May 1997

[13]. D. Wullich and L. Goldfeld. "Reduction of Peak Factor in orthogonal multi-carrier modulation by amplitude limiting and coding". IEEE Trans. Commun., vol 47, pp. 18-21, January 1999

[14]. D. Kim and G.L. StÜber. "Clipping nose mitigation for OFDM by decision- aided reconstruction". IEEE Commun. Letter, pp. 4-6, January 1999

[15]. C. Tellambura. "Use OFM-sequences for OFDM peakto-average power ratio reduction". Electronic Letter, vol. 33, no. 15 , pp. 1300-1301, July 1997

[16]. H.E. Rowe. "Memory less nonlinearities with Gaussian inputs: Elementary results". Bell Syst. Tech. J., vol. 61, no. 7, pp. 1519-1525, September 1982

[17]. G. Santella and F. Mazzenga. "A Hybrid analyticalsimulation procedure for performance evaluation in M-QAMOFDM schemes in presences of nonlinearity".

[18]. P. Banelli and S. Cacopardi. "Theoritical analysis and performance of OFDM signals in nonlinear AWGN channels". IEEE Trans. Commun., vol. 48, pp. 430-441, March 2000.

[19]. IEEE Standard for Local and Metropolitan Area Networks Part 16: Air Interface for Fixed Broadband Wireless Access Systems, IEEE Std. 802.16e-2005, 2005.

[20]. Hasan Zareian and Vahid Tabataba Vakili. "Analysis of NonLinear Distortion Using Orthogonal Polynomials HPA Model" IJCSNS, vol. 8, no. 1, January 2008

[21]. Hideki Ochiai and Hideki Imai. "Performance Analysis of Deliberately Clipped OFDM Signal" IEEE Trans. Commun., vol. 50, no. 1, January 2002

\section{BIOGRAPHIES}

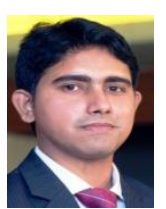

Mazid Ishtique Ahmed received his B.Sc. in Electrical and Electronic Engineering degree from Ahsanullah University of Science and Technology, Dhaka, Bangladesh on 2010. For following two years, he served as an It System Support and Solution Design Engineer in data edge limited, a system integrator and IT solution provider. $\mathrm{He}$ is in the position of Lecturer in Atish Dipankar University of Science and Technology and a graduate student in BRAC University, Bangladesh. His research interest includes Advanced Wireless
Communication Technology fields of LTE and OFDM system implementation with performance enhancement solutions.

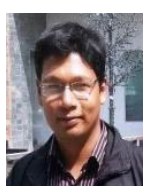

Chowdhury Muktadir Rahman received his B.Sc. in Electrical and Electronic Engineering degree from Ahsanullah University of Science and Technology, Dhaka, Bangladesh on 2010. Currently positioned as Specialist, PS Core Planning for Robi Axiata Limited, Bangladesh a renowned GSM operator. He contains 5 years of professional experience in IP technologies for GSM Carrier network in Mobile Backbone, GPRS, EDGE and HSPA+ technologies. He is an IP technology expert with various vendor certifications in the same field.

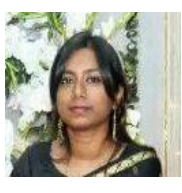

Sabiha Sattar completed her B.Sc. in Electrical and Electronic Engineering degree from Ahsanullah University of Science and Technology, Dhaka, Bangladesh on 2010. Currently positioned as Engineer, Electronics Division, Bangladesh Atomic Energy Center (BAEC). She is experienced and involved in Research and Development of Micro-controller based embedded system. 